\title{
Remarks on the impossibility of a Siegel-Shidlovskii like theorem for $G$-functions
}

\author{
T. Rivoal
}

\begin{abstract}
The Siegel-Shidlovskii Theorem states that the transcendence degree of the field generated over $\overline{\mathbb{Q}}(z)$ by $E$-functions solutions of a differential system of order 1 is the same as the transcendence degree of the field generated over $\overline{\mathbb{Q}}$ by the evaluation of these $E$-functions at non-zero algebraic points (expect possibly at a finite number of them). The analogue of this theorem is false for $G$-functions and we present conditional and unconditional results showing that any intermediate numerical transcendence degree can be obtained.
\end{abstract}

Keywords. Siegel-Shidlovskii Theorem, G-functions, logarithmic singularity

2010 Mathematics Subject Classification. 11J91, 34M35.

\section{Introduction}

In 1929, Siegel [Sie29] introduced two classes of arithmetic power series, $E$ and $G$-functions, which can be viewed as generalizations of $\exp (z)$ and $\log (1-z)$ respectively. An $E$-function is a formal power series $E(z)=\sum_{n=0}^{\infty} \frac{a_{n}}{n !} z^{n}$ such that the coefficients $a_{n}$ are algebraic numbers and there exists $C>0$ such that:

(i) the maximum of the moduli of the conjugates of $a_{n}$ is $\leq C^{n+1}$ for any $n$.

(ii) there exists a sequence of non-zero rational integers $d_{n}$, with $\left|d_{n}\right| \leq C^{n+1}$, such that $d_{n} a_{m}$ is an algebraic integer for all $m \leq n$.

(iii) $E(z)$ satisfies a homogeneous linear differential equation with coefficients in $\overline{\mathbb{Q}}(z)$.

Siegel's definition is slightly more general but it is believed that the distinction does not exists. Nowadays $E$-functions tend to be systematically defined as above. A $G$-function is a formal power series $G(z)=\sum_{n=0}^{\infty} a_{n} z^{n}$ satisfying $(i),(i i)$ and (iii) (with $G(z)$ instead of $E(z)$ there). Shidlovskii completed Siegel's results on the diophantine nature of $E$-functions; see [Shi89].

Theorem 1. (Siegel-Shidlovskii, 1929-1955) Let $Y(z)={ }^{t}\left(E_{1}(z), \ldots, E_{n}(z)\right)$ be a vector of $E$ functions solution of a differential system $Y^{\prime}(z)=A(z) Y(z)$ where $A(z) \in M_{n}(\overline{\mathbb{Q}}(z))$. Let $T(z)$ be the least common denominator of the entries of $A(z)$. Then for any $\alpha \in \overline{\mathbb{Q}}$ such that $\alpha T(\alpha) \neq 0$, we have

$$
\operatorname{deg} \operatorname{tr}_{\overline{\mathbb{Q}}(z)} \overline{\mathbb{Q}}(z)\left(E_{1}(z), \ldots, E_{n}(z)\right)=\operatorname{deg} \operatorname{tr}_{\overline{\mathbb{Q}}} \overline{\mathbb{Q}}\left(E_{1}(\alpha), \ldots, E_{n}(\alpha)\right) .
$$

Subsequent works by Nesterenko and Shidlovskii [NS96], André [And00, And15], Beukers [Beu06] gave a precise description of the polynomials relations when the transcendence degree is not maximal: basically, any numerical relation can be lifted to a functional one in the simplest way.

The diophantine theory of $E$-functions is thus essentially complete. This is far from true for $G$-functions, for which transcendence results are sporadic, let alone algebraic independence results. Following the pioneering works of Galochkin [Gal74], Bombieri [Bom79] and Chudnovsky [Chu84], the closest results in the direction of a Siegel-Shidlovskii like theorem for $G$-functions are of the following form.

We thank episciences.org for providing open access hosting of the electronic journal Hardy-Ramanujan Journal 
Theorem 2. (Chudnovsky, 1984) Let $Y(z)={ }^{t}\left(G_{1}(z), \ldots, G_{n}(z)\right)$ be a vector of $G$-functions solution of a differential system $Y^{\prime}(z)=B(z) Y(z)$, where $B(z) \in M_{n}(\overline{\mathbb{Q}}(z))$. Assume that

$$
\operatorname{deg} \operatorname{tr}_{\overline{\mathbb{Q}}(z)} \overline{\mathbb{Q}}(z)\left(G_{1}(z), \ldots, G_{n}(z)\right)=n .
$$

For any $d$, there exists $C=C(Y, d)>0$ such that, for any algebraic number $\alpha \neq 0$ of degree $d$ with $|\alpha|<\exp \left(-C \log (H(\alpha))^{\frac{4 n}{4 n+1}}\right)$, there does not exist a polynomial relation between $G_{1}(\alpha), \ldots, G_{n}(\alpha)$ over $\mathbb{Q}(\alpha)$ of degree $d$.

Here, $H(\alpha)$ is the naive height of $\alpha$. There is a long way between this statement and algebraic independence. In a special case, André [And96] proved a Siegel-Shidlovskii like theorem. His proof is a refinement of that of Chudnovsky.

Theorem 3. (André, 1996) For any $\alpha \in \overline{\mathbb{Q}}^{*}$ with $|\alpha|<1$, the two numbers ${ }_{2} F_{1}\left[\frac{1}{2}, \frac{1}{2} ; 1 ; \alpha\right]$ and ${ }_{2} F_{1}\left[-\frac{1}{2}, \frac{1}{2} ; 1 ; \alpha\right]$ are algebraically independent over $\overline{\mathbb{Q}}$.

These hypergeometric series form a solution of a differential system of dimension 2, and the theorem also has a $p$-adic counterpart. André's method, named simultaneous adelic uniformisation, is quite general in principle but has been applied so far only to the example described in the theorem, or to directly connected variations. The relation between these two hypergeometric series and periods of elliptic curves (and Jacobi theta functions) is crucial.

To the best of my knowledge, in the ring of $G$-values (see [FR14]), it is not yet known that there exist three numbers which are algebraically independent over $\overline{\mathbb{Q}}$, though we can find two which are algebraically independent over $\overline{\mathbb{Q}}$, for instance $\pi$ and $\Gamma(1 / 3)^{3}$, or $\pi$ and $\Gamma(1 / 4)^{4}$. Both results are highly non-trivial and due to Chudnovsky; they are also consequences of André's theorem.

However, the situation in this theorem does not describe the general case. Indeed, there exist many examples of transcendental $G$-functions $F(z)$ assuming algebraic values at some algebraic point $\alpha$. It is trivial that there exist examples with $\alpha \neq 0$ a singularity of the differential equation; indeed given a $G$-function $F(z)$ with $\alpha$ inside its disk of convergence, $\sqrt{1-z / \alpha} F(z)$ is also a $G$-function, but now $\alpha$ is a singularity of its minimal differential equation. But there also exist examples where $\alpha$ is not a singularity. The most striking ones are the "modular" hypergeometric series ${ }_{2} F_{1}(z)$, which takes algebraic values on a dense algebraic set (see [Arc03, Beu93, Wol88]), for instance ${ }_{2} F_{1}\left[\frac{1}{12}, \frac{5}{12} ; \frac{1}{2} ; z\right]$ is equal to $\frac{3}{4} \sqrt[4]{11}$ at $z=\frac{1323}{1331}$.

While giving a lecture on $E$ and $G$-functions where I presented the Siegel-Shidlovskii Theorem, I was asked to which extent it is impossible to prove an analogue for $G$-functions. The answer is probably part of the folklore but I could not find it, which led me to write this note.

Proposition 1. Given any non-zero distinct algebraic numbers $\alpha_{1}, \ldots, \alpha_{k}$ and any integer $n \geq 1$, there exists a vector $Y(z)={ }^{t}\left(G_{1}(z), \ldots, G_{n}(z)\right)$ of $G$-functions in $\mathbb{Q}[[z]]$, with $G_{n}(z)=1$, solution of a differential system $Y^{\prime}(z)=B(z) Y(z)$ where $B(z) \in M_{n}(\overline{\mathbb{Q}}(z))$ such that the following holds. Let $S(z)$ be the least common denominator of the entries of $B(z)$. Then

$$
\operatorname{deg} \operatorname{tr}_{\overline{\mathbb{Q}}(z)} \overline{\mathbb{Q}}(z)\left(G_{1}(z), \ldots, G_{n}(z)\right)=n-1,
$$

and, for all $j=1, \ldots, k$, we have $\alpha_{j} S\left(\alpha_{j}\right) \neq 0$ and

$$
\operatorname{deg} \operatorname{tr}_{\overline{\mathbb{Q}}} \overline{\mathbb{Q}}\left(G_{1}\left(\alpha_{j}\right), \ldots, G_{n}\left(\alpha_{j}\right)\right)=0
$$

Moreover, we can also prove results with other values for the numerical transcendence degree.

Proposition 2. For any integer $n \geq 2$, there exists a vector $Y(z)={ }^{t}\left(G_{1}(z), \ldots, G_{n}(z)\right)$ of $G$ functions in $\mathbb{Q}[[z]]$ solution of a differential system $Y^{\prime}(z)=B(z) Y(z)$ where $B(z) \in M_{n}(\overline{\mathbb{Q}}(z))$ such 
that the following holds. Let $S(z)$ be the least common denominator of the entries of $B(z)$. Then $S(1) \neq 0$,

and

$$
\operatorname{deg} \operatorname{tr}_{\overline{\mathbb{Q}}(z)} \overline{\mathbb{Q}}(z)\left(G_{1}(z), \ldots, G_{n}(z)\right)=n-1
$$

$$
\operatorname{deg} \operatorname{tr}_{\overline{\mathbb{Q}}} \overline{\mathbb{Q}}\left(G_{1}(1), \ldots, G_{n}(1)\right)=1 .
$$

We can replace the transcendence degree 1 by any integer in $\{2, \ldots, n-1\}$ provided we assume the (so far unproven) assumption that the numbers $\log (p)$, with $p$ running through the primes, are algebraically independent over $\overline{\mathbb{Q}}$.

Using the $G$-values $\pi$ and $\Gamma(1 / 3)^{3}$, we can obtain an unconditionnal result with numerical transcendence degree 2 .

Proposition 3. For any integer $n \geq 4$, there exists a vector $Y(z)={ }^{t}\left(G_{1}(z), \ldots, G_{n}(z)\right)$, with $G_{n}(z)=1$, of $G$-functions in $\overline{\mathbb{Q}}[[z]]$ solution of a differential system $Y^{\prime}(z)=B(z) Y(z)$ where $B(z) \in M_{n}(\overline{\mathbb{Q}}(z))$ such that the following holds. Let $S(z)$ be the least common denominator of the entries of $B(z)$. Then $S(1) \neq 0$,

$$
\operatorname{deg} \operatorname{tr}_{\overline{\mathbb{Q}}(z)} \overline{\mathbb{Q}}(z)\left(G_{1}(z), \ldots, G_{n}(z)\right)=n-1
$$

and

$$
\operatorname{deg} \operatorname{tr}_{\overline{\mathbb{Q}}} \overline{\mathbb{Q}}\left(G_{1}(1), \ldots, G_{n}(1)\right)=2 .
$$

All these results concern one algebraic point, or a finite number of algebraic points. It seems difficult to have small transcendence degree at infinitely many algebraic numbers for any $n \geq 3$ (the case $n=2$ is possible, due to Wolfart [Wol88]). It is a difficult problem to predict the polynomial relations between values of $G$-functions. The Bombieri-Dwork Conjecture implies that $G$-values should be, in some sense, periods of suitable algebraic varieties over $\overline{\mathbb{Q}}$; the converse is known to be true, by André's work in [And89]. Polynomial relations between periods are described by Grothendieck Conjecture, of which not much is known. Roughly speaking, it says that any polynomial relation between periods has a motivic origin. We refer to André's book [And04], especially Partie III from page 199.

The above results are not difficult to prove, provided we assume some well-known transcendence results. The real point is proving the algebraic independence over $\overline{\mathbb{Q}}(z)$ of the $G$-functions specially constructed to collectively assume few values at some given algebraic point. The easiest way for this is to use $G$-functions with logarithmic singularities at various points. An an illustration of the method, let us show that $\log (1-z)$ (defined in $D=\mathbb{C} \backslash[1, \infty)$ ) is not in $\overline{\mathbb{Q}}(z)$. Indeed, assume there exists $P \in \overline{\mathbb{Q}}[X, Y]$ such that $P(z, \log (1-z))=0$ for all $z \in D$; then by "turning" $m$ times around $z=1$, we get $P(z, \log (1-z)+2 i \pi m)=0$ for all $m \in \mathbb{Z}$. This implies that $P$ is a constant in the variable $Y$, ie that $P(X, Y)=P(X, 0)$; hence $P(z, 0)=0$ for all $z \in D$ which forces $P$ to be identically equal to 0 .

\section{Proof of Proposition 1}

The case $n=1$ is of no interest, we simply take $G_{1}(z)=1$. We now assume that $n \geq 2$. Let $P(z) \in \mathbb{Z}[[z]]$ of degree $\geq 1$ that does not vanish at $z=0$ and vanishing at $\alpha_{1}, \ldots, \alpha_{j}$. We define an integer $D \geq 1$ large enough such that for any $|z| \leq\left|\max _{\ell}\left(\alpha_{\ell}\right)\right|+1$, we have $\left|z^{k} P(z)\right|<D$. We now set

$$
G_{k}(z)=\log \left(1+z^{k} \frac{P(z)}{D}\right), \quad k=1, \ldots, n-1
$$

and $G_{n}(z)=1$. Each $G_{k}(z)$ can be expanded as a power series $\sum_{n=0}^{\infty} a_{k, n} z^{n} \in \mathbb{Q}[[z]]$ with radius of convergence $\geq\left|\max _{\ell}\left(\alpha_{\ell}\right)\right|+1$. In particular, the power series can all be evaluated at each $z=\alpha_{\ell}$, $\ell=1, \ldots, j$. 
Obviously, $G_{k}^{\prime}(z)=r_{k}(z) G_{n}(z)$ with

$$
r_{k}(z)=\frac{z^{k-1}\left(k P(z)+z P^{\prime}(z)\right)}{D+z^{k} P(z)}, \quad k=1, \ldots, n-1,
$$

so that $Y(z)={ }^{t}\left(G_{1}(z), \ldots, G_{n}(z)\right)$ is solution of a differential system $Y^{\prime}(z)=B(z) Y(z)$ with

$$
B(z)=\left(\begin{array}{cccc}
0 & \ldots & 0 & r_{1}(z) \\
0 & \ldots & 0 & r_{2}(z) \\
\vdots & \ldots & \vdots & \vdots \\
0 & \ldots & 0 & \left.r_{n-1(} z\right) \\
0 & \ldots & 0 & 0
\end{array}\right)
$$

Clearly, the $\alpha_{j}$ 's are not poles of any of the entries of $B(z)$ and the condition $\alpha_{\ell} S\left(\alpha_{\ell}\right) \neq 0$ is satisfied for each $\ell$.

Since $G_{n}\left(\alpha_{j}\right)=1$ and $G_{k}\left(\alpha_{\ell}\right)=0$ for $k=1, \ldots, n-1$ and $\ell=1, \ldots, j$, it remains to prove that

$$
\operatorname{deg} \operatorname{tr}_{\overline{\mathbb{Q}}(z)} \overline{\mathbb{Q}}(z)\left(G_{1}(z), \ldots, G_{n}(z)\right)=n-1 .
$$

In other words, we have to prove that there does not exist $Q \in \overline{\mathbb{Q}}\left[X_{1}, \ldots, X_{n}\right]$ such that

$$
Q\left(z, G_{1}(z), \ldots, G_{n-1}(z)\right)=0
$$

for all $z$ in $\mathbb{C}$ minus a certain number of cuts rooted at the zeroes of $1+z^{k} P(z) / D$, for $k=1, \ldots, n-1$. Let $\xi_{k}$ be any fixed root of $1+z^{k} P(z) / D$; clearly, $\xi_{k} \neq \xi_{j}$ if $k \neq j$. For any integer $m$, if we "turn" $m$ times around $\xi_{1}$, we obtain

$$
Q\left(z, G_{1}(z)+2 i \pi m, G_{2}(z) \ldots, G_{n-1}(z)\right)=0
$$

locally around $\xi_{1}$. As a polynomial in $X_{2}, Q\left(z, X_{2}, G_{2}(z) \ldots, G_{n-1}(z)\right)$ has infinitely many roots, hence it must be 0 in the variable $X_{2}$, which implies that

$$
0=Q\left(z, X_{2}, G_{2}(z) \ldots, G_{n-1}(z)\right)=Q\left(z, 0, G_{2}(z) \ldots, G_{n-1}(z)\right) .
$$

We now turn around $\xi_{2}$, and so on, to reach the conclusion that

$$
0=Q\left(z, X_{2}, X_{3} \ldots, X_{n}\right),
$$

for all $z$. Hence, $Q\left(X_{1}, \ldots, X_{n}\right)$ is in fact equal to $Q(0,0 \ldots, 0)=0$ and thus is identically zero.

\section{Proof of Proposition 2}

To get an example where $\operatorname{deg} \operatorname{tr}_{\overline{\mathbb{Q}}} \overline{\mathbb{Q}}\left(G_{1}(1), \ldots, G_{n}(1)\right)=1$, we take $G_{n}(z)=1, G_{k}(z)=\log (1-$ $\left.z^{k} / 2\right) \in \overline{\mathbb{Q}}[[z]]$ for $k=1, \ldots, n-1$. The same argument as in the proof of Proposition 1 gives $\operatorname{deg} \operatorname{tr}_{\overline{\mathbb{Q}}(z)} \overline{\mathbb{Q}}(z)\left(G_{1}(z), \ldots, G_{n}(z)\right)=n-1$, and for $k \geq 1, G_{k}(1)=-\log (2) \notin \overline{\mathbb{Q}}$ (unconditionally).

To get an example where $\operatorname{deg} \operatorname{tr}_{\overline{\mathbb{Q}}} \overline{\mathbb{Q}}\left(G_{1}(1), \ldots, G_{n}(1)\right)=\ell$ for a given integer $\ell \in\{2, \ldots, n-1\}$, we repeat the above arguments with $G_{n}(z)=1, G_{k}(z)=\log \left(1+\left(p_{k}-p_{k+1}\right) z^{k} / p_{k+1}\right) \in \overline{\mathbb{Q}}[[z]]$ for $k=1, \ldots, \ell-1$, and $G_{k}(z)=\log \left(1+\left(p_{\ell-1}-p_{\ell}\right) z^{k} / p_{\ell}\right) \in \overline{\mathbb{Q}}[[z]]$ for $k=\ell, \ldots, n-1$. Here, $p_{j}$ is the $j$-th prime number, from $p_{1}=2$. The functions are algebraically independent over $\overline{\mathbb{Q}}(z)$ and

$$
\operatorname{deg} \operatorname{tr}_{\overline{\mathbb{Q}}} \overline{\mathbb{Q}}\left(G_{1}(1), \ldots, G_{n}(1)\right)=\operatorname{deg} \operatorname{tr}_{\overline{\mathbb{Q}}} \overline{\mathbb{Q}}\left(\log \left(p_{1} / p_{2}\right), \ldots, \log \left(p_{\ell-1} / p_{\ell}\right)\right)=\ell
$$

conditionally. 


\section{Proof of Proposition 3}

Let us now construct a vector of $G$-functions leading to a numerical transcendence degree 2 . We start with the following result: the $G$-values $\pi$ and $\Gamma(1 / 3)^{3}$ are algebraically independent over $\overline{\mathbb{Q}}$. Let us consider the two $G$-functions

$$
f(z)=z_{2} F_{1}\left[1, \frac{1}{2} ; \frac{3}{2} ;-z^{2}\right]=\arctan (z)=\sum_{n=0}^{\infty}(-1)^{n} \frac{z^{2 n+1}}{2 n+1}
$$

and

$$
g(z)={ }_{2} F_{1}\left[\frac{4}{3},-\frac{1}{3} ; 1 ; \frac{z}{2}\right]=\sum_{n=0}^{\infty} \frac{\left(\frac{4}{3}\right)_{n}\left(-\frac{1}{3}\right)_{n}}{2^{n}(1)_{n}^{2}} z^{n} .
$$

Note that $f^{\prime}(z)=\frac{1}{1+z^{2}} \in \overline{\mathbb{Q}}(z)$.

We have $f(1)=\frac{\pi}{4}$ while $g(1) \in \frac{\pi}{\Gamma(1 / 3)^{3}} \overline{\mathbb{Q}}$. The latter relation follows from a general formula of Bailey [Sla66, p. 32, Eq. (1.7.1.8)]:

$$
{ }_{2} F_{1}\left[a, 1-a ; c ; \frac{1}{2}\right]=\frac{\Gamma\left(\frac{c}{2}\right) \Gamma\left(\frac{1+c}{2}\right)}{\Gamma\left(\frac{a+c}{2}\right) \Gamma\left(\frac{1+c-a}{2}\right)} .
$$

With $a=\frac{4}{3}$ and $c=1$, this gives

$$
g(1)=\frac{\Gamma\left(\frac{1}{2}\right)}{\Gamma\left(\frac{7}{6}\right) \Gamma\left(\frac{1}{3}\right)}=\frac{6 \Gamma\left(\frac{1}{2}\right)}{\Gamma\left(\frac{1}{6}\right) \Gamma\left(\frac{1}{3}\right)}=\frac{6 \Gamma\left(\frac{2}{3}\right)}{2^{2 / 3} \Gamma\left(\frac{1}{3}\right)^{2}}=4^{2 / 3} \sqrt{3} \frac{\pi}{\Gamma\left(\frac{1}{3}\right)^{3}},
$$

where we have used in succession three functional equations for the Gamma function (with $s=\frac{1}{6}$ and $\left.t=\frac{2}{3}\right)$ :

$$
\Gamma(s+1)=s \Gamma(s), \quad \Gamma(s) \Gamma\left(s+\frac{1}{2}\right)=2^{1-2 s} \Gamma\left(\frac{1}{2}\right) \Gamma(2 s), \quad \Gamma(t) \Gamma(1-t)=\frac{\pi}{\sin (\pi t)} .
$$

We also need to evaluate $g^{\prime}(1)$. By [Sla66, p. 15, Eq. (1.4.1.1)], we have

$$
g^{\prime}(z)=-\frac{2}{9}{ }_{2} F_{1}\left[\frac{7}{3}, \frac{2}{3} ; 2 ; \frac{z}{2}\right] .
$$

We can sum $g^{\prime}(1)$ by means of [Sla66, p. 32, Eq. (1.7.1.9)]:

$$
{ }_{2} F_{1}\left[a, b ; \frac{1+a+b}{2} ; \frac{1}{2}\right]=\frac{\Gamma\left(\frac{1}{2}\right) \Gamma\left(\frac{1+a+b}{2}\right)}{\Gamma\left(\frac{1+a}{2}\right) \Gamma\left(\frac{1+b}{2}\right)} .
$$

With $a=\frac{7}{3}, b=\frac{2}{3}$ and using the reflection formula (last identity in (4.1)), we obtain

$$
g^{\prime}(1)=-\frac{2 \Gamma\left(\frac{1}{2}\right) \Gamma(2)}{9 \Gamma\left(\frac{5}{3}\right) \Gamma\left(\frac{5}{6}\right)}=-\frac{1}{3} \sin \left(\frac{2 \pi}{3}\right) \sin \left(\frac{\pi}{6}\right) \frac{\Gamma\left(\frac{1}{3}\right) \Gamma\left(\frac{1}{6}\right)}{\Gamma\left(\frac{1}{2}\right)^{3}}=-\frac{\sqrt{3}}{2 \pi g(1)} .
$$

The point $z=1$ is not a singularity of the respective minimal differential equations of order 2 satisfied by $f(z)$ and $g(z)$. This is also the case for the functions $f\left(z^{k}\right)$ for any integer $k \geq 1$. For any $n \geq 4$, we now define $n G$-functions by $G_{k}(z)=f\left(z^{k}\right), k=1, \ldots, n-3, G_{n-2}(z)=g(z)$, $G_{n-1}(z)=g^{\prime}(z), G_{n}(z)=1$. The vector $Y(z)={ }^{t}\left(G_{1}(z), G_{2}(z), \ldots, G_{n}(z)\right)$ is solution of a differential system $Y^{\prime}(z)=M(z) Y(z)$ where the matrix $M(z) \in M_{n}(\overline{\mathbb{Q}}(z))$ is not singular at $z=1$.

By construction, we have

$$
\operatorname{deg} \operatorname{tr}_{\overline{\mathbb{Q}}} \overline{\mathbb{Q}}\left(G_{1}(1), \ldots, G_{n}(1)\right)=\operatorname{deg} \operatorname{tr}_{\overline{\mathbb{Q}}} \overline{\mathbb{Q}}\left(f(1), g(1), g^{\prime}(1)\right)=\operatorname{deg} \operatorname{tr}_{\overline{\mathbb{Q}}} \overline{\mathbb{Q}}\left(\pi, \Gamma\left(\frac{1}{3}\right)\right)=2 .
$$


Let us now prove that the functions $G_{k}(z), k=1, \ldots, n-1$, are algebraically independent over $\overline{\mathbb{Q}}(z)$. This is a consequence of the following facts. The singularities (at finite distance) of the functions $f(z)$ and $g(z)$ are $\pm i$ and 2 respectively. Moreover, locally around $z=i$,

$$
f(z)=\Phi_{1}(z) \log (i-z)+\Phi_{2}(z)
$$

for some $\Phi_{1}(z) \neq 0$ and $\Phi_{2}(z)$ holomorphic at $z=i$, while locally around $z=2$,

$$
g(z)=\Psi_{1}(z) \log (2-z)+\Psi_{2}(z)
$$

for some $\Psi_{2}(z)$ holomorphic at $z=2$ and $\Psi_{1}(z)=g(2-z) \neq 0$. Note that

$$
g^{\prime}(z)=\Psi_{1}^{\prime}(z) \log (2-z)+\Psi_{2}^{\prime}(z)+\frac{\Psi_{1}(z)}{z-2}
$$

also has infinite monodromy around $z=2$.

We start from an hypothetic polynomial relation

$$
Q\left(z, f(z), f\left(z^{2}\right), \ldots, f\left(z^{n-3}\right), g(z), g^{\prime}(z)\right)=0,
$$

for some non-zero polynomial $Q \in \overline{\mathbb{Q}}\left[X_{1}, \ldots, X_{n}\right]$. We first eliminate the functions $f\left(z^{k}\right)$ by "turning" around $e^{i \pi /(2 k)}, k=1, \ldots, n-3$. There remains a polynomial relation

$$
\widetilde{Q}\left(z, g(z), g^{\prime}(z)\right)=0 \text {. }
$$

To rule out such a relation, we cannot use the same argument as above because $g(z)$ and $g^{\prime}(z)$ have the same type of monodromy around $z=2$. However, they are algebraically independent over $\overline{\mathbb{Q}}(z)$ because the differential Galois group of the minimal differential equation satisfied by $g(z)$ is $S L_{2}(\mathbb{C})$ (see [BH89] for instance). Hence, the $G$-functions $G_{k}(z), k=1, \ldots, n-1$, are algebraically independent over $\overline{\mathbb{Q}}(z)$.

\section{References}

[And89] Y. André, G-functions and Geometry, Aspects of Mathematics 13, MPI, 1989.

[And96] Y. André, G-fonctions et transcendance, J. reine angew. Math. 476 (1996), 95-126.

[And00] Y. André, Séries Gevrey de type arithmétique I. Théorèmes de pureté et de dualité, Annals of Math. 151 (2000), 705-740.

[And04] Y. André, Une introduction aux motifs, Panorams et Synthèses 17, SMF, 2004

[And15] Y. André, Solution algebras of differential equations and quasi-homogeneous varieties: a new differential Galois correspondence, preprint 2011, to appear in Annales ENS.

[Arc03] N. Archinard, Exceptional sets of hypergeometric series, J. Number Theory 101 (2003), no. 2, $244-269$.

[Beu93] F. Beukers, Algebraic values of G-functions, J. reine angew. Math. 434 (1993) 45-65.

[Beu06] F. Beukers, A refined version of the Siegel-Shidlovskii theorem, Annals of Math. 163 (2006), no. 1, $369-379$.

[BH89] F. Beukers, G. Heckman, Monodromy for the hypergeometric function ${ }_{n} F_{n-1}$, Invent. math. 95.2 (1989), 325-354.

[Bom79] E. Bombieri, On G-functions, Recent progress in analytic number theory, Vol. 2 (Durham, 1979), pp. 1-67, Academic Press, London-New York, 1981.

[Chu84] G. V. Chudnovky, On applications of Diophantine approximations, Proc. Nat. Acad. Sci. U.S.A. 81 (1984), no. 22, Phys. Sci., 7261-7265.

[FR14] S. Fischler, T. Rivoal, On the values of G-functions, Commentarii Math. Helv. 29.2 (2014), 313-341.

[Gal74] A. I. Galochkin, Lower bounds of polynomials in the values of a certain class of analytic functions, Mat. Sb. (N.S.) 95 (137) (1974), 396-417, 471.

[NS96] Yu. V. Nesterenko, A. B. Shidlovskii, On the linear independence of values of E-functions, Mat. Sb. 187 (1996), no. 8, 93-108; translation in Sb. Math. 187 (1996), no. 8, 1197-1211.

[Sie29] C. L. Siegel, Über einige Anwendungen diophantischer Approximationen, Abh. Preuss. Akad. Wiss. 1, 1929. 
[Shi89] A. B. Shidlovskii, Transcendental Numbers, de Gruyter Studies in Mathematics 12, 1989.

[Sla66] L. J. Slater, Generalized Hypergeometric Functions, Cambridge University Press, 1966.

[Wol88] J. Wolfart, Werte hypergeometrischer Funktionen, Invent. math. 92 (1988) 187-216.

T. Rivoal

Institut Fourier

Université Grenoble 1, CNRS UMR 5582

100 rue des Maths, BP 74

38402 Saint-Martin d'Hères cedex

France

e-mail: Tanguy.Rivoal@ujf-grenoble.fr 